\title{
Using Charged Cosmic Ray Particles to Monitor the Data Quality of FACT
}

D. Hildebrand ${ }^{a}$, J. Adam $^{b}$, M. L. Ahnen ${ }^{a}$, D. Baack ${ }^{b}$, M. Balbo ${ }^{c}$, A. Biland ${ }^{* a}$,

M. Blank ${ }^{d}$, T. Bretz ${ }^{a, 1}$, K. Bruegge ${ }^{b}$, J. Buss ${ }^{b}, \mathbf{A}_{\text {. Dmytriiev }}{ }^{c}$, D. Dorner $^{d}$,

S. Einecke ${ }^{b}$, D. Elsaesser ${ }^{b}$, C. Hempfling ${ }^{d}$, T. Herbst ${ }^{d}$, L. Kortmann ${ }^{b}$, L. Linhoff ${ }^{b}$, M. Mahlke ${ }^{a, 1}$, K. Mannheim ${ }^{d}$, S. A. Mueller ${ }^{a}$, D. Neise ${ }^{a}$, A. Neronov ${ }^{c}$, M. Noethe ${ }^{b}$, J. Oberkirch ${ }^{b}$, A. Paravac ${ }^{d}$, F. Pauss ${ }^{a}$, W. Rhode ${ }^{b}$, B. Schleicher ${ }^{d}$, F. Schulz ${ }^{b}$,

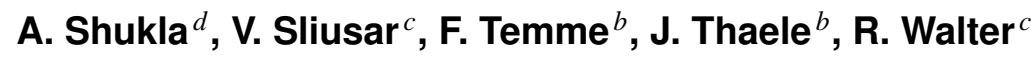

E-mail: dorothee.hildebrandephys. ethz.ch

${ }^{a}$ ETH Zurich, Institute for Particle Physics

Otto-Stern-Weg 5, 8093 Zurich, Switzerland

${ }^{b}$ TU Dortmund, Experimental Physics 5

Otto-Hahn-Str. 4, 44221 Dortmund, Germany

${ }^{c}$ University of Geneva, ISDC Data Center for Astrophysics

Chemin d'Ecogia 16, 1290 Versoix, Switzerland

${ }^{d}$ Universität Würzburg, Institute for Theoretical Physics and Astrophysics

Emil-Fischer-Str. 31, 97074 Würzburg, Germany

1 also at RWTH Aachen University

Imaging Air Cherenkov Telescopes (IACT) measure the faint flashes of Cherenkov light emitted by air-showers that are produced when charged particles or gamma rays hit the atmosphere. Therefore, the atmosphere above the IACT is an integral part of the detector. Variations in the performance of the IACT itself, but also changes in the absorption and scattering of Cherenkov light due to clouds or dust affect the interpretation of measured signals. Therefore, information about the status of the full system is crucial to combine measurements from different time periods. The First G-APD Cherenkov Telescope (FACT) is using for the first time solid state photosensors (so-called G-APDs or SiPM) to measure the flashes of Cherenkov light. Based on the stability of these sensors, we showed in the past that it is possible to identify the existence of strong clouds or calima when measuring the intrinsically constant flux of cosmic ray particles at different trigger levels. This necessitated dedicated measurements, preventing normal data taking in parallel. We have now improved the method to use instead those cosmic ray events that are recorded during normal data taking as dominant background. By applying a fixed virtual trigger threshold in software, we measure the rate of charged cosmic ray particles. A deviation from the expected flux allows to identify data sets with reduced performance of the complete system in quasi real-time, without the need for any additional device.

Applying the method to a data set when one of the 30 mirror tiles of FACT was missing, we show that a change of total yield of the Cherenkov light by few percent can be identified within few minutes of standard data taking. This nicely demonstrates that the hadron rate determined from standard data taking with FACT can be used for monitoring of the data quality.

35th International Cosmic Ray Conference - ICRC2017

10-20 July, 2017

Bexco, Busan, Korea

* Speaker.

(C) Copyright owned by the author(s) under the terms of the Creative Commons Attribution-NonCommercial-NoDerivatives 4.0 International License (CC B Y-NC-ND 4.0). 


\section{Introduction}

The First G-APD Cherenkov Telescope (FACT)[1] is pioneering the usage of solid state photo sensors (usually called SiPM or G-APD). The camera consists of 1440 pixels, each formed by a solid light guide and a $3 \times 3 \mathrm{~mm}^{2}$ sensor chip of type Hamamatsu MPPC S10362-33-50C. The data acquisition electronics is fully integrated into the camera body. It is mounted on a refurbished old HEGRA telescope with a mirror area of about $9.5 \mathrm{~m}^{2}$. Since the installation in October 2011 at the Canary Island La Palma, FACT is taking data whenever observation conditions permit.

When a high energetic charged cosmic ray particle or gamma-ray hits the atmosphere, it produces an extended air-shower consisting of several 1000 to billions of secondary particles, depending on the energy of the primary particle. Imaging Atmospheric Cherenkov Telescopes (IACT) like FACT observe the faint flashes of Cherenkov light emitted by these secondary particles. The evolution of the air-shower and the transmission of Cherenkov light is influenced by the atmosphere. Therefore, the atmosphere above the telescope is integral part of the detector.

An important step in analyzing IACT data is to check the data quality. Hardware issues with the telescope as well as clouds, dust and aerosols in the atmosphere can significantly alter the Cherenkov yield, i.e. the number of Cherenkov photons measured from a given air-shower. There exist several auxiliary devices that help to qualify the atmosphere, like cameras that monitor the extinction of star light or measure the number of visible stars to see clouds, or Pyrometers to measure the temperature of the sky to identify clouds. But it is not sufficient to identify e.g. the existence of a cloud above the IACT. A high altitude cloud can obscure the stars and change the sky temperature, but for air-showers evolving mainly below that cloud, the transmission of Cherenkov light is hardly affected. Lidars measure laser light reflected in different altitudes and are therefore better suited to qualify the atmosphere. Nevertheless, they have the drawback that the photosensors of IACT cameras are also sensitive to this laser light. In the case of FACT, the high rate of fake events induced by the nearby Lidar of MAGIC [3] can saturate the readout electronics. Therefore, a special electronics had to be developed by MAGIC and FACT to veto the FACT trigger in case of a laser shot. For a large IACT array like the future CTA[4], where individual sub-arrays might point towards different sky regions, the necessity for operation of several Lidars can be challenging.

\section{The Method}

Instead of using auxiliary devices to monitor the data quality, we develop a passive method that is based only on standard IACT data taking. The flux of high energy cosmic ray particles in the TeV energy range detectable by FACT is known to be constant and isotropic[5]. Therefore, the measured rate is expected to be constant for a given pointing direction of the telescope. As we have shown in [6], performing so-called trigger ratescans are an ideal tool to monitor the performance of the complete system. For this, the rate of triggered events is recorded versus the trigger threshold. Setting the threshold high enough, the amount of accidental triggers induced by photons of the night sky background can be neglected and the measured rate is only due to air-showers induced by high energetic charged cosmic ray particles ${ }^{1}$. A significant reduction in the measured trigger

\footnotetext{
${ }^{1}$ The amount of charged cosmic ray particles is orders of magnitude higher than the amount of gamma-rays even when observing the brightest sources. Therefore, gamma-ray contamination is negligible.
} 
rate therefore indicates a reduced Cherenkov yield. This can be identified in quasi-real time. The main issue with this method is that (at least with the trigger electronics of FACT) it is necessary to interrupt data taking to perform such a ratescan, needing precious observation time. Therefore, we have improved the method to use only standard data taking. While the electronics of FACT does not deliver detailed trigger information for each individual event, it is easy possible to reconstruct this. The FACT trigger is a simple analogue sum of the signal amplitude of nine adjacent pixels [1]. A similar adding can be done for a recorded event and the maximum amplitude $A_{\max }$ been searched in this event ${ }^{2}$. This event would have been recorded for any trigger setting $A_{\text {trig }}$ less than $A_{\max }$. It is now possible to emulate a virtual ratescan, using all the recorded events of a data set and counting for different virtual trigger setting how many events have an $A_{\max } \geq A_{\text {trig }}$.

\section{Choosing the Virtual Trigger Threshold}

It is crucial to keep the performance of the camera as stable as possible. As shown in [2], the FACT camera is self-calibrating and can be kept very stable without the need for any auxiliary calibration device. It is only necessary to adjust the voltage applied to the sensors to their temperature and DC current.

$A_{\text {trig }}$ should be chosen high enough that accidental triggers are negligible under all observational conditions, but low enough to have a reasonably high counting rate to reduce statistical fluctuations. For this study, we use $A_{\text {trig }}=700 \mathrm{mV}$, corresponding to about 70 recorded photons on nine adjacent pixels within few $\mathrm{ns}^{3}$.

All data taken with FACT from the main observation targets (Crab Nebula, Mrk421, Mrk501, 1ES1959) until end of 2016 fulfilling very soft cuts are used in this study. These cuts are:

- Exclude data taken before June 2014, since there were minor modifications in the calculation of the voltage to be applied to the sensors before March 2014. This results in slightly different gain and therefore different $A_{\max }$ for a given event. Data in March/April 2014 were taken with same voltage conditions, but the reflector was missing one out of 30 mirror tiles.

- Exclude runs with a duration less than 4 minutes. Normal data runs have a duration of 5 minutes, except during twilight operations where duration of a run is limited to 1 minute to better handle the very fast variation of ambient light conditions. This cut removes the twilight runs and the few runs that were aborted due to data taking problems.

- Exclude runs with a deadtime $>15 \%$. This removes mainly those runs affected by MAGIC Lidar when the veto was not operational ${ }^{4}$. Deadtimes are taken into account when calculating rates.

Figure 1 shows the virtual trigger rate for $A_{\text {trig }}=700 \mathrm{mV}$ versus the zenith angle of the observation. A clear band is visible that represents the standard performance of the detector. Individual

\footnotetext{
${ }^{2}$ Since the readout electronics of FACT can introduce rare spikes, $A_{\max }$ is requested to have a duration of at least two nanoseconds.

${ }^{3}$ The signal shape in has during about $3 \mathrm{~ns}$ an amplitude $>95 \%$ of the maximum amplitude calibrated to $10 \mathrm{mV}$ [2].

${ }^{4}$ FACT readout has an intrinsic deadtime less than $0.3 \%$, but it can reach several $\%$ if the MAGIC Lidar has to be vetoed and can fully saturate while the Lidar is operated without veto.
} 
runs have significantly reduced rate, indicating a reduced Cherenkov yield. The red curve shows a preliminary cut to indicate bad runs below the line. The shape of the curve is based on an educated guess and the zenith dependence of the cut is taken as

$$
4.5 \times\left(\cos (1.1 \times z)+1.1 \times \sin (2 \times z)^{3}\right)
$$

with $z$ being the zenith distance in radians ${ }^{5}$.

Up to a zenith distance of $\approx 40^{\circ}$, there is no strong zenith dependence visible. To not spoil remaining plots shown in this study with data where the expected standard rate is reduced due to zenith dependence, runs having a zenith distance $>40^{\circ}$ are excluded from now on.

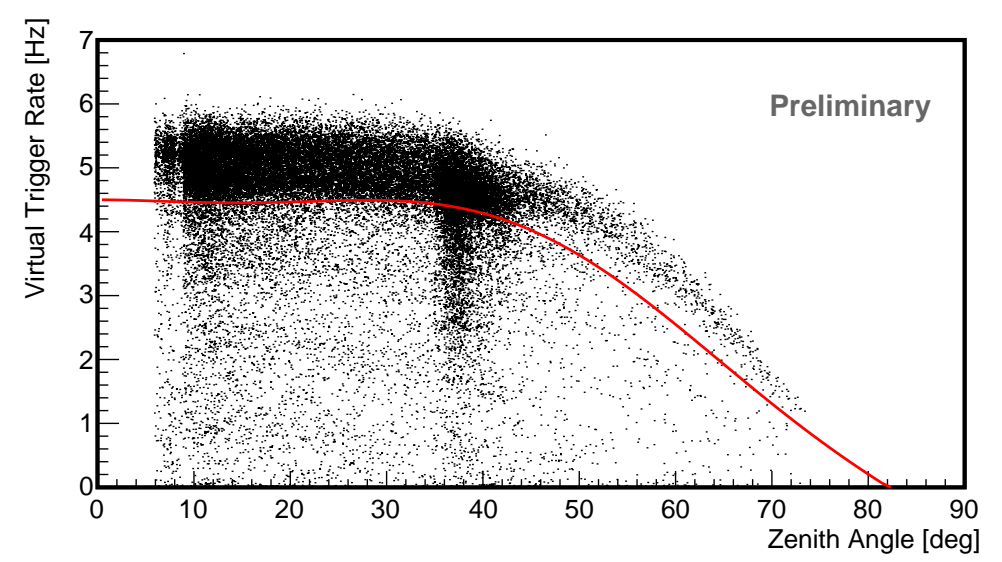

Figure 1: Distribution of the virtual trigger rate in dependence of the zenith distance. Each point corresponds to a five minute run. The red line is a preliminary cut to distinguish between good and bad runs. The source $1 \mathrm{ES} 1959$ culminating $\approx 35^{\circ}$ seems strongly affected by worse atmospheric conditions.

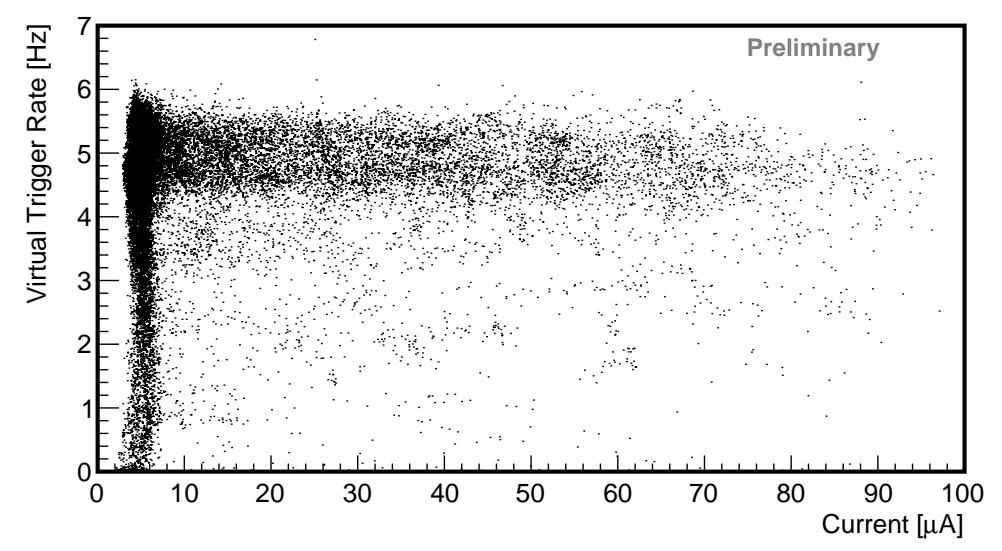

Figure 2: Distribution of the virtual trigger rate against the DC current that is correlated with the amount of ambient light. Each dot corresponds to a five minute run measured with a zenith distance $<40^{\circ}$.

\footnotetext{
${ }^{5} \mathrm{~A}$ better description of the shape based on scientific assumptions can be found in [7].
} 
Figure 2 shows the virtual trigger rate for $A_{\text {trig }}=700 \mathrm{mV}$ versus the DC current drawn by the photo sensors. This current is proportional to the amount of photons recorded, so mainly a measure of the amount of ambient light. During dark nights, the current is $\approx 5 \mu \mathrm{A}$ and corresponds to a measured background rate of $\approx 30$ million photons per second and pixel. FACT standard operation extends to a current of at most $100 \mu \mathrm{A}$, corresponding to more than 500 million photons per second and pixel. Most data is taken under dark conditions, i.e. no moon light or pointing far from the moon. But also for data with current $\gg 10 \mu \mathrm{A}$ the dark band of standard rate shows no significant dependency from the amount of ambient light. Therefore, a cut based on the virtual trigger rate for $A_{\text {trig }}=700 \mathrm{mV}$ does not have to include a dependency on the DC current.

\section{Long Term Behavior}

Plotting the virtual trigger rate versus MJD (Figure 3), there is a clear band of data with good performance. Runs with significantly reduced rate can be identified. On top of this, there is a longterm seasonal structure visible. Investigations are ongoing to fully characterize and understand this systematics ${ }^{6}$, but this is beyond the scope of this presentation. Nevertheless, temperature dependency of the SiPM sensors can be excluded as a major contribution [10]. As long as this systematics cannot be predicted, rather loose cuts should be applied to decide about data quality in real time. For offline analysis, the shape can be taken into account and a harder cut be applied.

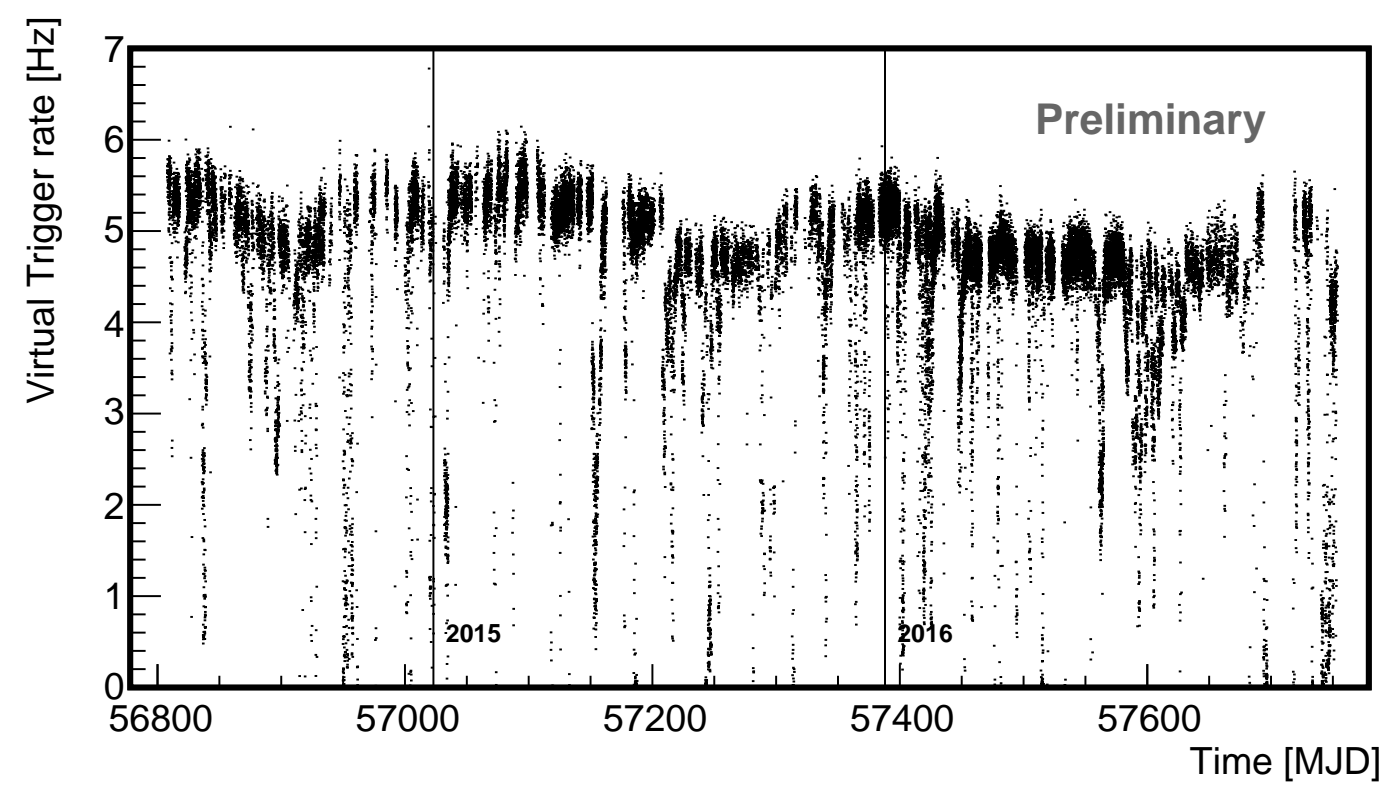

Figure 3: Distribution of the virtual trigger rate versus time for 2.5 years. Short term reduction of the rate indicate reduced Cherenkov yield. The reason for the seasonal variation is under investigation.

\footnotetext{
${ }^{6}$ Seasonal variations due to change of the atmosphere are predicted by [8], and an effect mainly due to aerosols has been reported in [9].
} 


\section{Sensitivity of the Method}

There remains the question to what reduction of Cherenkov yield the method is sensitive to. This can be investigated with extensive Monte Carlo studies, but preliminary information can also be gained by comparing the virtual trigger rates recorded in early 2014. In March/April 2014, one of the 30 mirror tiles of the FACT reflector was missing after being damaged in a heavy snow storm. This corresponds to an $\approx 3.3 \%$ reduction of the Cherenkov light focused into the camera. Comparing the distributions in Figure 4 one can see an $\approx 13 \%$ reduction of the virtual trigger rate with reduced mirror area compared to the rates measured after mirror repair.

Taking into account the integral spectral in-

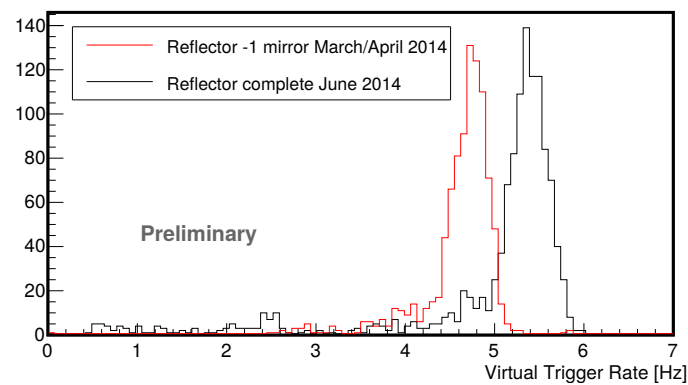

Figure 4: Distribution of the virtual trigger rate measured during and after one of the 30 mirror tiles of the FACT reflector was missing. dex of charged cosmic ray particles of $\approx-1.7$ [5], from a 3.3\% reduction in Cherenkov yield a corresponding rate reduction of $\approx 6 \%$ is expected. There could be an additional contribution from the seasonal variation, but (at least for 2015 and 2016) this effect has the tendency to result in lower rates measured in summer. The reason for the observed further rate reduction is under investigation ${ }^{7}$. Nevertheless, this measurement and [10] indicate that even with a small IACT like FACT, a reduction of the Cherenkov yield on the percentage level can be seen within few minutes.

\section{Applying the Method}

The Crab nebula is expected to emit a constant flux of gamma-rays and is therefore used as a standard candle. To check if the method does reliably identify data sets with reduced data quality, it would be best to apply it to observations of the Crab nebula and check if runs with a small reconstructed flux get rejected. But FACT with it's small mirror area is mainly sensitive to rare gamma-rays with energies in the $\mathrm{TeV}$ energy range. This results in measuring only few tens of gamma-rays from the Crab nebula per hour,

Instead, we use the so-called background rate. This is the number of hadronic events misidentified as gamma-rays but not originating from the direction of the observed source. To have the lowest energy threshold, only observations during dark nights and a zenith distance $<40^{\circ}$ are taken into account. To increase the statistics for individual data sets, four sequential five minute runs are grouped into a twenty minutes run.

Figure 5 shows the background rate calculated for each data set with the Quick Look Analysis in quasi real-time[11]. Runs plotted in red are those that are excluded by the cut used in figure 1. It is evident that runs with lowest rates are cut. Due to large statistical fluctuations and a cut that already rejects runs with moderate reduction of the virtual trigger rate, it is not expected that all rejected runs show significantly reduced background rate.

\footnotetext{
${ }^{7}$ During the repair, all mirrors were realigned. In first approximation, this should not affect a sum-trigger study since the light is still focused into a trigger patch. But there might be higher order effects.
} 


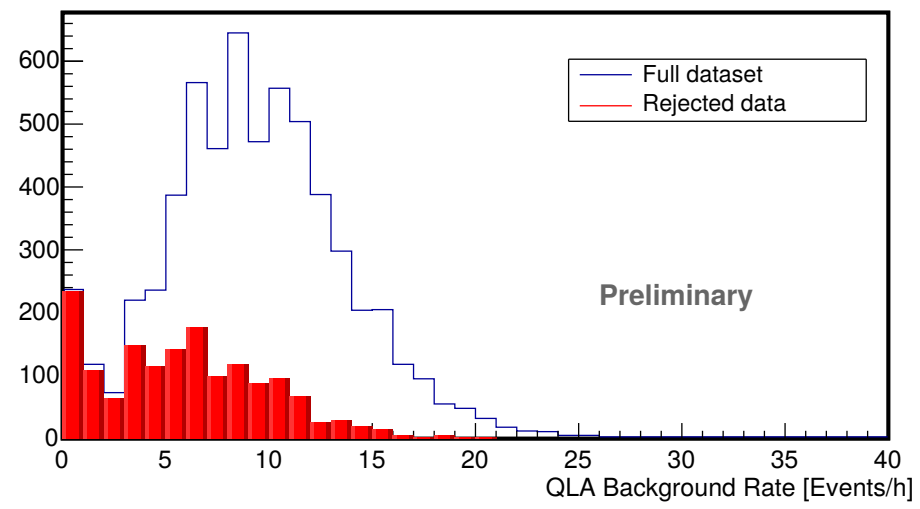

Figure 5: Distribution of the background rate calculated with the Quick Look Analysis of FACT for data sets taken during dark night and zenith distance $<40^{\circ}$. The red entries are removed when applying the cut shown in figure 1

\section{Summary and Outlook}

We have presented a method that allows to qualify data taken with FACT in quasi real time without the need for any auxiliary device or dedicated observation time. While more work is necessary to fully understand all effects, it is evident that within few minutes a small telescope like FACT can identify a change of the Cherenkov yield.

We can imagine several application for this method at IACTs, among them:

- It allows to measure data taking quality in quasi real time without the need to operate auxiliary devices like a Lidar. This can simplify operation and reduce costs. If a Lidar is still preferred due to the possibility to characterize the atmosphere in detail, the method allows to reduce the operation time of the Lidar: When the virtual trigger rate is good, the atmosphere is good and there is no need for Lidar measurements. If the virtual trigger rate is bad, there are too strong atmospheric effects to be reliably corrected for. So a Lidar is only necessary in case of moderate reduction of the virtual trigger rate. This results in less disturbance of neighboring instruments.

- Since the method is sensitive to the performance of the complete system consisting of the telescope and the atmosphere, it can also indicate reduced performance of a telescope itself. Long term degradation or significantly differing measurements between neighboring telescopes will be a strong indication for a hardware problem of one telescope.

Due to the steep power law spectrum of cosmic ray particles, large IACTs that are sensitive to also lower energetic showers are able to work with higher counting rates and therefore better sensitivity. In addition, higher energetic showers penetrate deeper into the atmosphere and the majority of Cherenkov photons are emitted from the shower maximum close to the end. So higher energetic and therefore brighter showers are less affected by high altitude clouds than lower energetic ones. So for large IACTs, it seems feasible to apply different $A_{\text {trig }}$ values. Clouds at rather high altitude would then only affect the virtual trigger rates for small $A_{\text {trig }}$, while low altitude clouds would affect the rates for all $A_{\text {trig }}$ values. This would allow to gain information about altitude distribution of atmospheric effects. 
Even more precise information can probably be gained by not using only trigger-like information as in the presented method. By fully reconstructing each hadronic event one can extract the spectrum of charged cosmic ray particles. Alterations of the measured spectra between datasets could contain sufficient information about the atmosphere to extract and apply correction factors.

As mentioned before, there is no need for any auxiliary device or for dedicated observation time to apply this method.

\section{Acknowledgments}

The important contributions from ETH Zurich grants ETH-10.08-2 and ETH-27.12-1 as well as the funding by the Swiss SNF and the German BMBF (Verbundforschung Astro- und Astroteilchenphysik) and HAP (Helmoltz Alliance for Astro- particle Physics) are gratefully acknowledged. Part of this work is supported by Deutsche Forschungsgemeinschaft (DFG) within the Collaborative Research Center SFB 876 "Providing Information by Resource-Constrained Analysis", project C3. We are thankful for the very valuable contributions from E. Lorenz, D. Renker and G. Viertel during the early phase of the project. We thank the Instituto de Astrofisica de Canarias for allowing us to operate the telescope at the Observatorio del Roque de los Muchachos in La Palma, the Max-Planck-Institut für Physik for providing us with the mount of the former HEGRA CT3 telescope, and the MAGIC collaboration for their support.

\section{References}

[1] H. Anderhub et al. (FACT Collaboration), Design and operation of FACT - the first G-APD Cherenkov telescope, JINST 8 P06008 (2013)

[2] A. Biland et al. (FACT Collaboration), Calibration and performance of the photon sensor response of FACT - the first G-APD Cherenkov telescope, JINST 9 P10012 (2014)

[3] C. Fruck et al., A novel LIDAR-based Atmospheric Calibration Method for Improving the Data Analysis of MAGIC, Proceedings of the $33^{\text {rd }}$ ICRC, Rio de Janeiro, 2013

[4] M.Actic et al. (CTA Consortium), Design concepts for the Cherenkov Telescope Array CTA: An advanced facility for ground-based high-energy gamma-ray astronomy, Exper.Astron. 32 (2011)

[5] C. Patrignani et al. (Particle Data Group), The Review of Particle Physics, Chin. Phys. C, 40, 100001 (2016)

[6] D. Hildebrand et al. (FACT Collaboration), FACT - Measuring Atmospheric Conditions with Imaging Air Cherenkov Telescopes, Proceedings of the $33^{\text {rd }}$ ICRC, Rio de Janeiro, 2013

[7] M.Mahlke et al. (FACT Collaboration), FACT - Searching for periodicity in five-year light-curves of Active Galagic Nuclei, these proceedings

[8] K. Bernloehr, Impact of atmospheric parameters on the atmospheric Cherenkov technique, Astropart. Phys. 12 (2000)

[9] J. Hahn et al., Impact of aerosols and adverse atmospheric conditions on the data quality for spectral analysis of the H.E.S.S. telescopes, Astropart. Phys. 54 (2014)

[10] D. Hildebrand et al. (FACT Collaboration), Higher Order Temperature Dependence of SiPM used in FACT, these proceedings, ID 778

[11] D. Dorner et al. (FACT Collaboration), FACT - Monitoring Blazars at Very High Energies, Proceedings of the 2014 Fermi Symposium 